\title{
PREVALENCIA DE FLUOROSIS DENTAL EN ESCOLARES DE 6 A 15 AÑOS DE EDAD DE LA ZONA URBANA DE BUCARAMANGA
}

${ }^{1}$ Sonia Constanza Concha $S,{ }^{2}$ Yelena Celedón, ${ }^{2}$ Waldina Vera, ${ }^{2}$ Eliana Poveda,${ }^{2}$ Claudí Muñoz,${ }^{2}$ Tatiana Vergel, ${ }^{2}$ Diana Luna, ${ }^{2}$ Luz Miyelán Rodríguez, ${ }^{2}$ Constanza Arteaga, ${ }^{3}$ Diana Marina Camargo L.

${ }^{1}$ Odontóloga, Especialista en Educación y Comunicación para la Salud y el Bienestar, Docente U. Santo Tomás,

${ }^{2}$ Estudiantes de X semestre, Facultad de Odontología, U. Santo Tomás, ${ }^{3}$ M Sc Epidemiología, Profesora auxiliar Escuela de Fisioterapia, U. Industrial de Santander

Autor responsable de correspondencia. Dra. Sonia Constanza Concha S.

Correo electrónico: sococosa@yahoo.com

\section{RESUMEN}

Objetivo: Determinar la prevalencia de fluorosis dental en escolares entre 6 y 15 años de edad, de instituciones educativas públicas y privadas, del área urbana de Bucaramanga.

Materiales y métodos: Se seleccionaron 196 escolares entre los 6 y 15 años de edad, en forma aleatoria, mediante un muestreo por conglomerados. Se consideró la edad, el género, el tipo de institución educativa a la que estaban vinculados y el nivel TFI en cada molar, premolar e incisivo superior, de acuerdo a los criterios de Thylstrup y Fejeskov. Se analizaron las posibles asociaciones entre edad, género y tipo de institución con la prevalencia de fluorosis, y se consideró como unidad de análisis el sujeto y la estructura dentaria, aplicando la prueba de $\mathrm{Chi}^{2}$ y el test exacto de Fischer, con un nivel de significancia $\alpha=0.05$.

Resultados: Se evaluaron en 28 conglomerados, 196 escolares entre los 6 y los 15 años de edad y 1562 dientes, el 77\% con IC 95\% (69.6\% - 84.4\%) de los pacientes registraron espectros de fluorosis entre TFI 0 a 1 y TFI 0 a 6 . Los pacientes que registraron espectros de fluorosis entre TFI 0 a 3, fueron los de mayor frecuencia en un 29.1\% de los examinados con IC 95\% (19.37\% 38.8\%). La prevalencia de fluorosis fue mayor en el grupo de 9 a 11 años $87.2 \%(\mathrm{p}=0.001)$ y en instituciones públicas $94 \%$ ( $\mathrm{p}$ $=0.03$ ). Al análisis por diente, se encontró que el $52.8 \%$ de los dientes examinados registraron fluorosis. Hubo diferencias estadísticamente significativas al analizarlos por edad, con una proporción del $77.6 \%$ en el grupo de 6 a 8 años ( $p<0.000)$, y por institución educativa, del 42.8\%; en colegios privados en el grupo de 12 a 15 años ( $p=0.006$ ), y del 69.1\% en colegios públicos en el grupo de 9 a 11 años $(\mathrm{p}<0.001)$. Al analizar por tipo de diente, se encontró que los incisivos centrales fueron los más frecuentemente afectados con TFI 1 en el $25.8 \%$ de los pacientes. El diente más severamente afectado fue el primer premolar con TFI 3 en el 15.3\% de la muestra.

Conclusiones: Se registró una prevalencia de fluorosis del 77\% y una proporción del 52.8\% de dientes con fluorosis, en los escolares de 6 a 15 años matriculados en instituciones públicas y privadas; sin embargo, su severidad muestra que en los niveles de TFI 0 está el 47\% de los dientes y TFI de 1 a 3 en el 48\% de los dientes. [Concha SC, Celedón Y, Vera W, Muñoz C, Vergel T, Luna D, Rodríguez LM, Arteaga C, Camargo DM. Prevalencia de fluorosis dental en escolares de 6 a 15 años de edad de la zona urbana de Bucaramanga. Ustasalud Odontologia 2003: 2: 73 - 82]

PALABRAS CLAVE: Fluorosis, prevalencia, índice TF, escolares, flúor.

Dental fluorosis prevalence in 6 to 15 years old scholars from the city of Bucaramanga.

ABSTRACT

Purpose: To establish dental fluorosis prevalence in 6 to 15 years old students, registered in public and private schools of Bucaramanga.

Material and methods: A cluster randomized sampling to select the institution. Age, gender, and TF index by Thysltrup and Fejerskov criteria were considered. Associations with gender, age and institution were evaluated by patient and dental structure applying $\mathrm{X}^{2}$ and Fisher exact test considering $\alpha=0.05$.

Results: Twenty-eight clusters were analyzed. One hundred ninety six children, 6 to 15 years old with 1562 teeth. Dental fluorosis prevalence was 77\%, CI 95\% (69.6\% - 84.4\%), TFI range between TFI 0 - 1 to TFI 0 - 6 . The patients with TFI 0 to 3 were the highest frequency in $29.1 \%$, CI 95\% (19.37\% - 38.8\%). The prevalence in 9 to 11 years old group was $87.2 \%$, and in the public schools $94 \%$, both statically significant $(\mathrm{p}=0.001,0.003)$, respectively. The analyses by tooth showed fluorosis in $52.8 \%$ of teeth.

Conclusions: In 6 to 15 years Bucaramanga scholars, the fluorosis prevalence was 77\%, and 52.8\% teeth had fluorosis; however, the severity level in teeth was TFI level 0 (without fluorosis) 47\% and TFI 1 to 3, 48\%.

KEY WORDS: Dental fluorosis, prevalence, scholars, fluor, TF index 


\section{INTRODUCCIÓN}

La caries y la enfermedad periodontal continúan como problemas de salud pública en Colombia y en muchos países en desarrollo. ${ }^{1.4}$ Según el ENSAB III, en Colombia, la proporción de caries a los 7 años es de un 73.8\%; la pérdida de la inserción periodontal afecta al 32.8\% de las personas de 15 a 19 años, y $87 \%$ de las personas mayores de 55 años. ${ }^{2}$ Una de las estrategias más utilizadas en la prevención de la caries dental es el empleo de los fluoruros en sus diversas presentaciones..$^{2-4}$

Dentro de las estrategias para promocionar la salud dental y prevenir la caries cabe destacar, en primera instancia, las orientadas a modificar el medio ambiente oral, como la educación en hábitos higiénicos y dietéticos, el control del flujo salival y la elevación del contenido iónico de calcio, fósforo y flúor en la saliva. En segundo lugar, están las acciones dirigidas a erradicar el agente infeccioso, como el uso de antimicrobianos, reducir la transmisión de microorganismos, la modificación de la dieta y el uso de fluoruros. Finalmente, se consideran las acciones destinadas al fortalecimiento del huésped, entre las que se podrían destacar la colocación de sellantes de fosas y fisuras y la administración de fluoruros. ${ }^{4}$

Los fluoruros se constituyen en una alternativa preventiva muy versátil. El efecto preventivo de los compuestos fluorurados se fundamenta en su capacidad de favorecer la mineralización, acelerar la remineralización y modificar el metabolismo de los microorganismos cariogénicos ${ }^{4 \cdot 6}$ Este compuesto químico puede administrarse a las personas en forma tópica y/o sistémica logrando reducir la prevalencia de caries. ${ }^{4-6}$

Desde mediados del siglo pasado, Dean y otros investigadores han centrado su interés, en el efecto de los fluoruros administrados en forma sistémica, particularmente en el agua, para la prevención de la caries dental, y por otro lado, en la determinación de los niveles óptimos de fluoruros, vital para evitar la dentofluorosis, condición asociada a la ingesta de fluoruros. ${ }^{1,7}$

Dean definió la fluorosis dental o dentofluorosis como la hipomineralización del esmalte o la dentina, por la excesiva ingesta de fluoruros durante la formación dentaria. Murray y Moller la definen como un disturbio específico de la formación dentaria por la ingesta excesiva de fluoruros durante la odontogénesis, y Fejerskov como un defecto del desarrollo del esmalte inducido por los fluoruros. ${ }^{8}$ Otros autores la han definido como el indicador clínico tardío de una intoxicación sistémica por flúor acumulado, durante el periodo del desarrollo y/o maduración de los diente afectados. ${ }^{9}$

Diferentes artículos registran un aumento de la prevalencia de fluorosis en un nivel mundial, ${ }^{8-13}$ lo cual ha generado controversia sobre el uso de los fluoruros y la necesidad de controlar el consumo y vigilancia de sus efectos. ${ }^{9 \cdot 12} \mathrm{La}$ medición de esta condición se ha realizado mediante diversos índices, dentro de los que cabe destacar el índice de Dean (DI), el índice de fluorosis por superficie dental (TSIF), el índice de riesgo de fluorosis (FRI) y el índice de Thylstrup - Fejerskov (TFI). Cada uno de los cuales tiene sus opositores y sus seguidores, lo que ha dificultado la comparación entre los estudios. ${ }^{14}$

El DI ha sido propuesto por la OMS como una alternativa para resolver esta situación; ${ }^{15}$ sin embargo, en la literatura se han encontrado cuestionamientos en la forma de medición de la fluorosis (especialmente por la categoría de dudoso) y en la forma de expresar los resultados (como el promedio ponderado de los dientes con fluorosis). ${ }^{16,17}$ Otra alternativa, ampliamente usada, es el TFI, que ha mostrado según lo expresado por sus autores y otros investigadores, la virtud de medir la severidad y permitir la correlación de los signos clínicos con la rasgos histológicos. ${ }^{6,14,16,17}$

Hoy en Colombia, la preocupación por el posible incremento de la fluorosis y su probable asociación con la adición del flúor a la sal, ha aumentado el interés por vigilar los niveles de fluoruros y su posible efecto sobre el esmalte. ${ }^{9,18-20}$ Los estudios sobre fluorosis dental realizados muestran cómo en el nivel nacional hay una prevalencia del $11.5 \%$, de acuerdo con lo reportado en el ENSAB III. El índice empleando fue el DI. ${ }^{2}$ Otros estudios, en Manizales, empleando el DI, mostraron una prevalencia de $98 \%{ }^{21}$ En Bogotá, un estudio realizado en 660 escolares, entre los 5 y los 9 años, mostró una prevalencia $74.5 \%{ }^{9,22}$ Se desconoce la prevalencia de fluorosis en Bucaramanga, por lo cual el objetivo de este estudio fue determinar la prevalencia de fluorosis dental en escolares entre 6 y 15 años de edad de instituciones educativas públicas y privadas del área urbana de Bucaramanga.

\section{4}




\section{MATERIALES Y MÉTODOS}

Se realizó un estudio observacional descriptivo, ${ }^{23}$ cuya población de estudio estuvo conformada por escolares de ambos géneros, en planteles educativos públicos y privados de Bucaramanga, que cumplieron los siguientes criterios: Ser nacido y procedente de la zona urbana de Bucaramanga y estar entre los 6 y 15 años de edad cumplidos. Se excluyeron los escolares con compromiso sistémico, que afectara la apariencia clínica del esmalte, con alteraciones mentales o con aparatología oral, que impidiera la observación clínica, y finalmente aquellos niños cuyos padres, colegio o ellos mismos, rehusaran participar en el estudio.

En el momento del examen se excluyeron los dientes cariados, fracturados y obturados en más de las dos terceras partes de la superficie vestibular, los dientes con cálculos en esta superficie y los dientes con menos de $4 \mathrm{~mm}$ de corona clínica erupcionada.

La población de estudio estuvo representada por 70.000 escolares entre 6 y 15 años de edad, residentes en el municipio de Bucaramanga. La muestra fue seleccionada mediante un muestreo por conglomerados con base en una prevalencia esperada de $15 \%$ de fluorosis, ${ }^{1}$ un margen de error de $5 \%$ y un $95 \%$ de confianza.

El muestreo por conglomerado consistió en la selección aleatoria de 28 instituciones, de un total de 50 concentraciones educativas que aceptaron participar en el estudio, en cada uno de los cuales se examinaron 7 individuos al azar, que cumplieron con los criterios de inclusión, para un total de 196 sujetos. ${ }^{24}$

Se recolectaron las variables sociodemográficas generales como edad, género y el carácter público y privado de la institución escolar. El índice para cuantificar la fluorosis fue el TFI, ya que este índice es más sensible y preciso comparado con el DI; además, registra en escala ordinal los cambios histopatológicos encontrados en los dientes examinados, clasificándolos en 10 grados, de 0 a 9 (donde 0 representa la ausencia de fluorosis y 9 el nivel más severo), y refleja la severidad de los defectos en forma ascendente. ${ }^{17}$ El examen se efectuó en molares, premolares e incisivos del arco superior y se excluyeron los caninos.

Para el examen clínico se seleccionaron y estandarizaron 4 evaluadores. Se aplicaron los criterios del manual de fluorosis dental para trabajadores de la salud, ${ }^{17}$ bajo la dirección de una profesional experta en fluorosis. Se enfatizó en el diagnóstico diferencial fundamentado en el área dental afectada, forma, delimitación, color, dientes involucrados y otras pigmentaciones no fluoróticas. ${ }^{17,25}$

Los resultados de la estandarización revelaron una buena concordancia intraevaluadores con valores de kappa de $0.71,0.87,0.91$ y 0.94 para cada uno de los evaluadores respectivamente. El análisis de concordancia entre evaluadores presentó un coeficiente de correlación intraclase de $0.75 .^{26,27}$

El examen se llevó a cabo en los colegios previamente seleccionados de la siguiente manera: A los escolares se les realizó la limpieza de los dientes por medio de cepillado manual; luego el examinador secó los dientes con rollos de algodón. El examen intraoral se realizó por medio de la inspección visual bajo luz natural y un espejo intraoral y finalmente los resultados fueron registrados en un formulario para cada individuo. ${ }^{17}$

La base de datos se elaboró en el programa Excel por duplicado; ${ }^{28}$ se exportó al programa Epi-info 6.2 y mediante la rutina Validate se verificó la calidad de la digitación. ${ }^{29}$ Posteriormente se exportó al paquete Stata 6.0, para su análisis definitivo. ${ }^{30}$

La evaluación de las posibles asociaciones entre edad, género y tipo de institución con la prevalencia de fluorosis por individuo y diferentes dientes afectados se realizó aplicando la prueba de $\mathrm{Chi}^{2}$ y el test exacto de Fisher. El análisis consideró un nivel de significancia $\alpha=0.05$. ${ }^{24,31}$

\section{RESULTADOS}

El total de pacientes evaluados fue 196 y los dientes examinados 1562. La población estuvo en un rango de edad de 6 a 15 años con un mediana de 11 años y un promedio de edad de 10.4 años \pm 2.5 años

La prevalencia de fluorosis, por severidad, se resume en la Tabla 1. El 77\% de los pacientes IC 95\%, (69.6\% - 84.4\%) registraron fluorosis con espectros del TFI 0 a 1 al TFI 0 a 6 (definiendo espectro como todos posibles niveles de TFI que podrían ser observados en los dientes de un paciente examinado). Los pacientes que registraron espectro de 
fluorosis entre el TFI 0 a 3, fueron los de mayor frecuencia en un $29.1 \%$ con IC $95 \%$ (19.37\% - 38.8\%) del total de los sujetos examinados.

La prevalencia de fluorosis fue ligeramente superior en mujeres $77.8 \%$ (77) pero no hubo diferencia estadísticamente significativa ( $\mathrm{p}=0.804)$; la diferencia por edad fue estadísticamente significativa $(\mathrm{p}=0.001)$; la mayor prevalencia se observó en el grupo de 9 a 11 años $87.2 \%$ (68) seguido por el grupo de 6 a 8 años $81.6 \%$ (40). En cuanto a categoría de colegio en el análisis crudo se observó que no había diferencia estadísticamente significativa ( $\mathrm{p}=0.062$ ) (Tabla 2); pero al estratificar por edad se observó que había diferencias estadísticamente significativas $(p=0.03)$ entre colegio público y privado en el grupo de 9 a 11 años, siendo mayor la proporción en colegios públicos $94 \%$ (47) (Tabla 3).
Tabla 1. Frecuencia de fluorosis según severidad.

\begin{tabular}{lll}
\hline TFI & Fluorosis & $\%$ \\
0 & 45 & 23 \\
$0-1$ & 29 & 14.8 \\
$0-2$ & 37 & 18.9 \\
$0-3$ & 57 & 29.1 \\
$0-4$ & 22 & 11.2 \\
$0-5$ & 5 & 2.5 \\
$0-6$ & 1 & 0.5 \\
\hline $\mathrm{n}=196$ & &
\end{tabular}

Tabla 2. Prevalencia de fluorosis según género, grupo etáreo y categoría del colegio.

\begin{tabular}{|c|c|c|c|}
\hline \multirow[b]{2}{*}{ Variable } & \multicolumn{3}{|c|}{ Prevalencia de fluorosis } \\
\hline & $\begin{array}{l}\text { Si } \\
N^{0} \quad \% \\
N=151\end{array}$ & $\begin{array}{l}\text { No } \\
\mathrm{N}^{\circ} \quad \% \\
\mathrm{~N}=45\end{array}$ & $\mathrm{P}^{*}$ \\
\hline $\begin{array}{l}\text { Género } \\
\text { Mujeres }\end{array}$ & $77(77.8)$ & $22(22.3)$ & \\
\hline Hombres & $74(76.3)$ & $23(23.7)$ & 0.804 \\
\hline $\begin{array}{l}\text { Grupo etá } \\
\text { (años) } \\
6-8\end{array}$ & $40(81.6)$ & $9(18.4)$ & \\
\hline $9-11$ & $68(87.2)$ & $10(12.8)$ & \\
\hline $12-15$ & $43(62.3)$ & $26(37.7)$ & 0.001 \\
\hline $\begin{array}{l}\text { Típo de in } \\
\text { Público }\end{array}$ & $81(82.6)$ & $17(17.4)$ & \\
\hline Privado & $70(71.4)$ & $28(28.6)$ & 0.062 \\
\hline
\end{tabular}


Tabla 3. Prevalencia de fluorosis según tipo de institución estratificado por grupo etáreo.

\begin{tabular}{|c|c|c|c|c|c|}
\hline Grupo etáreo & \multicolumn{2}{|c|}{ Público \% } & \multicolumn{2}{|c|}{ Privado $\%$} & $\mathrm{P}^{*}$ \\
\hline $6-8$ & 23 & $(85.2)$ & 17 & (77.3) & 0.48 \\
\hline $9-11$ & 47 & (94) & 21 & (75) & $0.03^{* *}$ \\
\hline $12-15$ & 11 & (52.3) & 32 & $(66.6)$ & 0.26 \\
\hline $\begin{array}{l}\text { Póblicas: } 6 \\
\text { Privados: }\end{array}$ & 8 & $12-15$ a & & "Chir & le fisher \\
\hline
\end{tabular}

Tabla 4. Distribución del TFI analizado por diente.

\begin{tabular}{lccc} 
Nivel TFI & Dientes con Fluorosis $\%$ & \\
TFI & 0 & 738 & 47.2 \\
TFI & 1 & 293 & 18.8 \\
TFI & 2 & 244 & 15.6 \\
TFI & 3 & 213 & 13.6 \\
TFI & 4 & 66 & 4.2 \\
TFI & 5 & 7 & 0.5 \\
TFI & 6 & 1 & 0.06 \\
\hline $\mathrm{n}=1562$ & & &
\end{tabular}

La frecuencia de fluorosis analizada por dientes mostró que el $52.8 \%$ de los dientes examinados presentaban fluorosis entre TFI 1 y TFI 6. Entre los dientes que presentaban fluorosis, el TFI 1 fue el que predominó en el 18.8\% (293) de los dientes; la proporción de dientes con fluorosis fue ligeramente superior en hombres $(53.7 \%)$, pero no fue estadísticamente significativa $(p=0.46)$. La diferencia por edad fue estadísticamente significativa $(\mathrm{p}<0.000)$ observándose una mayor frecuencia de dientes afectados por fluorosis en el grupo de 6 a 8 años 77.6\%(149), seguido por el grupo de 9 a 11 años $60.7 \%$ (383).
En cuanto a la categoría de colegio analizada por dientes examinados, el análisis crudo muestra diferencias estadísticamente significativas $(\mathrm{p}<0.001)$ entre colegios públicos y privados (Tabla 5). Al estratificar por edad se observaron diferencias estadísticamente significativas ( $\mathrm{p}=0.006$ ). En el grupo de 12 a 15 años, el análisis mostró una mayor proporción de fluorosis en los colegios privados; para el grupo de 9 a 11 años también se observó una diferencia estadísticamente significativa $(p<0.001)$ entre colegios públicos y privados siendo mayor la prevalencia en colegios públicos (Tabla 6).

El análisis de la distribución intraoral de la fluorosis permite observar que el nivel del índice TF, que se presentó con mayor frecuencia en cualquier tipo de diente fue el TFI 0 (ausencia de fluorosis); su frecuencia fue mayor en los dientes de erupción temprana, como los centrales con $45.9 \%$, el primer molar con $44.6 \%$ y el lateral con $41.3 \%$. El TFI 1 y 2 se presentó con mayor frecuencia en centrales laterales y primeros molares, con 38.8\%, 33.4\% y 30.9\%, respectivamente. El TFI 3 y 4 se presentó más en el primer premolar (19.9\%), seguido del segundo premolar (18.1\%), primer molar (13.7\%) y finalmente segundo mo$\operatorname{lar}(7.4 \%)$.

El TFI 5 y 6 se presentó con mayor frecuencia en incisivos centrales con $0.6 \%$. Los dientes que con más frecuencia se excluyeron de ser examinados, fueron los segundos molares, segundo y primeros premolares con una proporción de 74\%, 50\%, y 43.9\%, respectivamente. 
Tabla 5. Frecuencia de fluorosis por dientes examinados según género, grupo etáreo y categoria del colegio.

Prevalencia de fluorosis

$\begin{array}{llll}\text { Variable } & \mathrm{Si} & \mathrm{No} & \mathrm{P}^{*} \\ & \mathrm{~N}^{\circ} \quad \% & \mathrm{~N}^{\circ} \quad \% & \\ \mathrm{~N}=824 & \mathrm{~N}=738 \%\end{array}$

Género

Mujeres

$430(51.9)$

$399(48.1)$

Hombres

$394(53.7)$

$339(46.3)$

0.46

Grupo etáreo

(años)

$\begin{array}{lllll}6-8 & 149 & (77.6) & 43 & (22.4)\end{array}$

$\begin{array}{llll}9-11 & 383 & (60.7) & 248\end{array}$

$\begin{array}{llllll}12-15 & 292 & (39.5) & 447 & (60.5) & 0.000\end{array}$

Tipo de institución

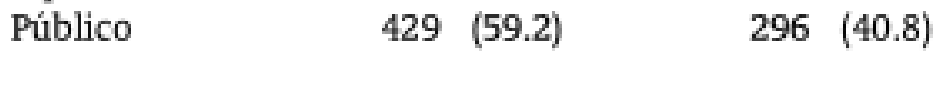

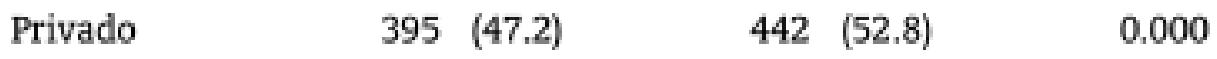

$\mathrm{n}=1562$

Tabla 6. Frecuencia de fluorosis por dientes examinados según tipo de institución estratificado por grupo etáreo

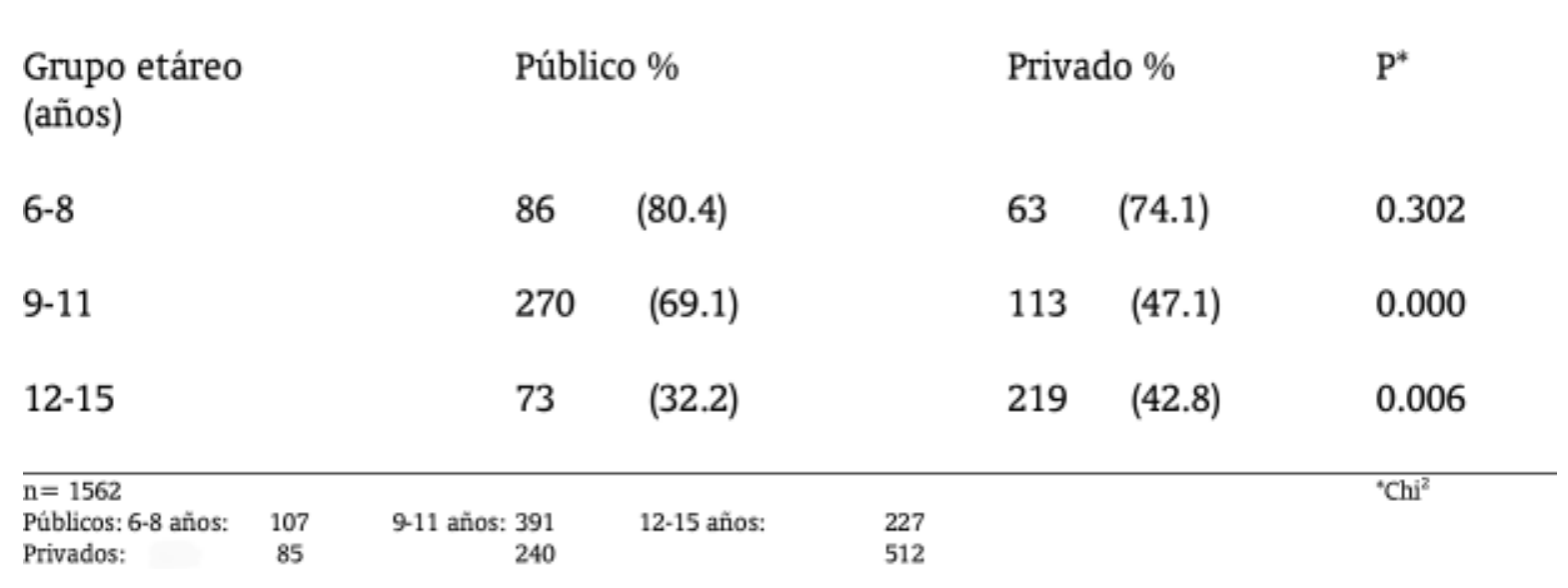




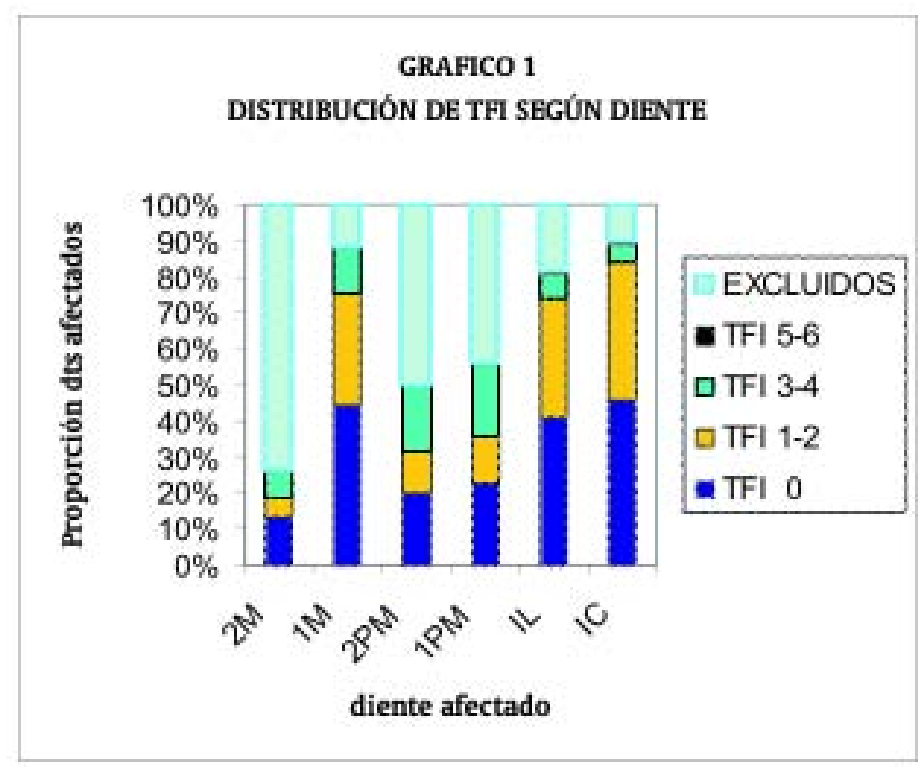

\begin{tabular}{lcccccc}
\hline \multicolumn{1}{c}{ TFITIP DTE } & 2M & IM & 2PM & IPM & IL & IC \\
TFI 0 & 13,3 & 44,6 & 19,9 & 23 & 41,3 & 45,9 \\
TFI 1-2 & 5,4 & 30,9 & 11,5 & 13,0 & 33,4 & 38,8 \\
TFI 3-4 & 7,4 & 13,0 & 18,1 & 19,9 & 6,9 & 4,6 \\
TFI 5-6 & 0,0 & 0,3 & 0,0 & 0,3 & 0,3 & 0,6 \\
EXCLUIDOS & 74,0 & 11,2 & 50,0 & 43,9 & 18,4 & 10,2 \\
\hline
\end{tabular}

La condición de excluido es más frecuente en los dientes de erupción tardía (segundo molar, segundo premolar y primer premolar). El TFI 0 (ausencia de fluorosis) es una condición más frecuente para los dientes de erupción temprana (central, primer molar y lateral) (Gráfico 1).

\section{DISCUSIÓN}

La prevalencia total de fluorosis en los escolares de instituciones públicas y privadas de Bucaramanga, sobre un total de 196 estudiantes examinados, fue de un $77 \%$ con IC 95\% (69.6\% - 84.43\%); sin embargo, es importante destacar que, el $23 \%$ de los sujetos no registraron fluorosis (TFI 0), en el 33.7\% de los sujetos examinados su espectro de fluorosis estaría en TFI 0 a 1 y TFI 0 a 2, que serían equiparables a formas leves de fluorosis de acuerdo al ID y en un $40.3 \%$ de los examinados su espectro de fluorosis estaría en TFI 0 a 3 y TFI 0 a 4, que al compararlo con el índice de Dean, se hablaría de formas moderadas, según lo expresado por Fejerskov y colaboradores. ${ }^{17}$
Dean, en 1936, reportó que con el uso continuo de agua, cuyo contenido ideal de flúor fue $1 \mathrm{ppm}$, era posible que se desarrollaran las formas más leves de fluorosis dental en aproximadamente el 10\% de un grupo de sujetos examinados. En aguas, en las que el contenido es de 1.7 a 1.8 ppm de flúor, la prevalencia puede elevarse al 40 ó 50\%, y la distribución porcentual de la severidad, será de la forma leve o muy leve. ${ }^{19}$ Sin embargo, aquí surge el problema de los catalogados como dudosos, de acuerdo con el indice de Dean, pues es probable que algunas de las formas de TFI 1 e incluso TFI 2, pudiesen catalogarse como dudosas de acuerdo con el ID y surgieran, por tanto los problemas al comparar estos resultados con las conclusiones establecidas por este autor.

No todos los dientes en una boca están igualmente afectados por la fluorosis dental. El grado al cual los diferentes grupos de dientes en una boca están afectados, es uno de los rasgos característicos de la fluorosis dental. En el presente estudio, este aspecto que es considerado por Thylstrup y Fejerskov como criterio fundamental para el 
diagnóstico de fluorosis, fue considerado a través de lo definido como espectro de fluorosis permitiendo así reconocer en un paciente desde la ausencia hasta las formas más severas del fenómeno en estudio.

Los resultados de esta investigación, coinciden con lo reportado por Thylstrup y otros autores quienes encontraron que los dientes menos afectados por fluorosis son los incisivos y los primeros molares, mientras que los premolares y segundos molares permanentes son los más severamente afectados. ${ }^{17}$

La fortaleza del TFI en estudios poblacionales es que permite conocer la prevalencia de fluorosis y cuantificar la frecuencia de esta alteración del esmalte en cada uno de los espectros. El análisis de los espectros permite conocer el nivel de severidad alcanzado en cada paciente, sin dar espacio al nivel de dudoso; adicionalmente, se puede analizar el comportamiento por estructura dentaria y dar una mayor riqueza al análisis. Al emplear en este estudio un índice para cuantificar fluorosis con estas características, se está tratando de dar mayor validez y riqueza en el análisis, aspectos que son importantes a la hora de presentar resultados.

Donde el examen se ha llevado a cabo en niños entre los 69 años, los resultados indican una baja prevalencia y severidad de fluorosis dental. Cuando los examinados son niños entre los 11 y 15 años de edad, los premolares y los segundos molares están más severamente afectados. ${ }^{17}$

El patrón puede modificarse ligeramente en áreas con altos niveles de flúor como en aquellas que registran niveles que superan $1.2 \mathrm{ppm}$, y la población exhibe formas severas de fluorosis dental. En tales áreas, el primer molar registra una severidad de fluorosis similar a la de los premolares y segundos molares permanentes. ${ }^{17}$

Los datos del presente estudio muestran patrones de severidad similares a los de zonas con bajos niveles de flúor, suministrados sistémicamente, en los que a pesar de observarse una frecuencia relativamente alta de fluorosis en los incisivos, las formas más severas se observan en los dientes con erupción tardía, que para este estudio fueron las formas de TFI 3 y 4; sin embargo esta afirmación es necesario hacerla con cautela para segundos molares, pues una elevada proporción de estos que no pudieron ser examinados.
Según Fejerskov, mediante el uso del TFI ha sido posible registrar, en forma lógica, los rasgos clínicos de la fluorosis dental sin riesgo de confusión, con los cambios inducidos en el esmalte, por elementos diferentes de los fluoruros. ${ }^{17}$ Fraysse y Poueza, por su parte, reportan que sólo la presencia de estroncio en el agua de beber ha hecho que se induzcan ciertos tipos de esmalte moteado; ${ }^{16}$ que aunado a criterios de diagnóstico diferencial claros, podría llevarnos a pensar que las lesiones observadas en el presente estudio son realmente lesiones fluoróticas y no otro tipo de lesiones.

En cuanto a las formas de TFI hasta 5 y 6 , que representan el $3 \%$ del total de la prevalencia, sus causas no pueden explicarse a través de este estudio, pues en ningún momento se intentó establecer relaciones de causalidad. En estos grupos en particular, se podría pensar en otras de fuentes adicionales de flúor, que podrían estar representadas en cremas dentales, con elevadas concentraciones de fluroruros, que pudieron ser ingeridas, ${ }^{13,17}$ pues la tendencia general observada, es el uso de una crema para toda la familia y generalmente para adultos.

Otra posible fuente podrían representarla los enjuagatorios; pero se desconoce la frecuencia de su uso en este grupo particular, durante el proceso de la formación dentaria. ${ }^{17,32}$ Las fuentes nutricionales serían una alternativa. No se descarta la presencia de problemas sistémicos, del tipo de alteración renal, desórdenes metabólicos o respiratorios, nivel de desnutrición y el consumo de ciertos medicamentos, ${ }^{33}$ generalmente asociados a las alteraciones del equilibrio-ácido básico, especialmente en las formas acidóticas. ${ }^{17}$

La fluoruración del agua o la sal, aun en niveles óptimos, causa fluorosis dental; en consecuencia habría que definir si biológicamente esto es aceptable en términos de prevalencia y severidad y que niveles de fluorosis estaría el gobierno, el gremio odontológico y la comunidad dispuesto a permitir. ${ }^{19}$

Los datos reportados en la ciudad de Bogotá, fueron del $74.7 \%$, usando TFI; lo que en cierta medida, permitiría afirmar que se registran niveles de prevalencia que son semejantes entre en Bogotá y Bucaramanga, pues en esta última fue $77 \% .^{22}$

En Manizales se reportó una prevalencia del 98\%, pero es dificil de comparar por el hecho de haberse utilizado el índice de Dean. ${ }^{21}$ Además en esa ciudad se administró

80

Ustasalud Odontología 2003; 2: 73 - 82

Concha SC. y col. 
flúor a través del agua, mucho antes de la fluoruración de la sal, lo cual estaría mostrando niveles de fluorosis relacionadas con otras fuentes.

El posible sesgo de selección, relacionado con la edad en el presente estudio, se refleja en el hecho de que la mayor proporción de escolares de las concentraciones públicas se encontraban en el grupo de 9 a 11 años (51\%); en colegios privados, la mayor proporción de sujetos examinados estaba en el grupo de 12 a 15 años, que representaba el 48\% de esta población (no se muestra); sin embargo, esto se corrigió categorizando por fluorosis, por institución educativa y según el grupo etáreo (Tabla 3).

De acuerdo con lo anterior se puede concluir que en Bucaramanga se registra una prevalencia de fluorosis del 77\% con IC 95\% (69.6\% - 84.4\%) en los escolares de 6 a 15 años de instituciones públicas y privadas del área urbana de Bucaramanga; sin embargo, la fluorosis es evidente primordialmente en dientes de erupción temprana y con espectros de fluorosis de TFI entre el 0 y 3 . Es probable que la administración sistémica de flúor, dentro de las que se encuentra la sal, pueda haber ejercido alguna influencia en los cambios de los patrones de presencia de fluorosis. Sin embargo, estos no parecen alejarse de los esperados para áreas que están sometidas a una medida masiva controlada de fluororuración de tipo sistémica y en la que los niveles de flúor en el agua no han sobrepasado el 1 a $1.2 \mathrm{ppm}^{34}$ y que para la sal serían de 180 a 220 ppm.

Es necesario desarrollar nuevas investigaciones sobre este mismo grupo poblacional con el fin de determinar el comportamiento de la fluorosis sobre los dientes excluidos, representados por aquellos de erupción tardía, probablemente más afectados en cantidad y severidad. Sería fundamental monitorear las posibles fuentes sistémicas de fluoruros y determinar qué alteraciones sistémicas podrían estar modificando esta condición; un estudio de muestras de sal, en Bucaramanga, mostró cómo el 40\% de las muestras analizadas registraban niveles inferiores a los reglamentados, por lo que se podría presumir que la sal no es la única fuente sistémica de flúor. ${ }^{35}$

Nuevas investigaciones orientadas a monitorear las causas, a analizar el comportamiento de la fluorosis sobre los diferentes grupos etáreos, a educar a los padres, profesores y escolares sobre la ingesta de fluoruros particularmente en dentífricos y el uso de cremas dentales pediátricas aportarian nuevas evidencias y orientarían las políticas de la salud pública, en particular, la de fluoruración de la sal. Podrían proveer datos concluyentes alrededor de esta medida de salud y ayudar a tomar las mejores decisiones en beneficio de los individuos y de la comunidad, pues esta medida, en particular, ha generado una álgida discusión y aún no hay respuestas definitivas sobre esta medida de salud pública.

\section{Agradecimientos.}

A la odontóloga Estefanía Martignon como profesional experta que apoyó el proceso de estandarización y sus generosos aportes a fin de lograr un trabajo con la mejor calidad posible.

\section{BIBLIOGRAFÍA}

1. Thylstrup A, Fejerskov 0. Textbook of Clinical Cariology. 2nd. Ed. Copenhague: Ed. Munksgaard; 1994.

2. Estudio Nacional de Salud Bucal-ENSAB III. Colombia: Ministerio de Salud. Tomo VII, 1999.

3. Axelsson P. An introduction to risk prediction and preventive dentistry. Chicago: Quintessence Publishing Co; 1999.

4. Axelsson P. Diagnosis and Risk Prediction of Dental Caries. Chicago: Quintessence Publishing Co; 2000.

5. Nikiforuk G. Caries Dental. Aspectos básicos y clínicos. Paraguay: Editorial Mundi; 1986.

6. Seif T. Cariología. Prevención, diagnóstico y tratamiento contemporáneo de la caries dental. Caracas: Actualidades Médico-Odontológicas Latinoamérica; 1997.

7. Dean H, Trendley O. Classification of mottled enamel diagnosis. Jour A.D.A. 1934: 1421 - 1426.

8. Curtes TW. Suckling GW. Differential Diagnosis of Dental Fluorosis. J Dent Res 1990; 714 - 719.

9. Galindo F. La fluorosis dentaria nueva enfermedad de la niñez colombiana. Revista de la Academia Colombiana de Odontología Pediátrica. 1998; 2 : 5 - 7.

10. Villa AE, Guerrero S, Icaza G, Villalobos J, Anabolon M. Dental fluorosis in Chilean children: evaluation of risk factors. Community Dent Oral Epidemiol 1998; 26: 310 - 315.

11. Ibrahim YE, Bjorvatn K, Birkeland JM. Caries and dental fluorosis in a 0.25 and 2.5 ppm fluoride area in the Sudan. Internacional Journal of Paediatric Dentistry 1997; 7: 161 - 166. 
12. Kumar JV, Swango PA, Lininger LL, Leske GS, Green EL, Haley VB. Changes in dental fluorosis and dental caries in Newburgh and Kingston. American Journal of Public Health 1998; 88: 1866 - 1870.

13. Adair SM, Mcknight Hanes C, Russell CM, Whitford GM. Dental caries and fluorosis among children in a rural Georgia area. Pediatr Dent 1999; 21: 81 - 85.

14. Kingman A. Current techniques for measuring dental fluorosis: Issues in data analysis. Adv Dent Res 1994; 8: 5665.

15. Organización Mundial de la Salud. Encuesta de Salud Bucodental. Métodos básicos. Cuarta edición. 1997 p.35.

16. Fraysse C, Pouezat JA. Relevance of epidemiological indices for assessing dental fluorosis Wld helth quart 1994; 47: 6264.

17. Fejerskov O, Firoze M, Baelum V, Mller IJ. Dental fluorosis a handbook for health workers Munsksgaard; 1988.

18. Tovar S. Dentro de los compromisos de la odontología en la salud pública: Vigilancia del uso de los fluoruros. Revista de la Federación Odontológica Colombiana 2001; 60: 33 - 46.

19. González MC. Efectos sistémicos del flúor en Colombia. Revista de la Academia Colombiana de Odontología Pediátrica 1998; 2: 10 - 14.

20. Segura MJ, Bermúdez EM. Descripción y análisis del sistema de vigilancia epidemiológica del programa de fluoruración de la sal en Colombia. Revista de la Federación Odontológica Colombiana 19982000; 57 - 72 .

21. Sánchez H. Fluoruros en la salud pública. Universidad Autónoma de Manizales 1989; 1: 7 - 28.

22. Granados OL. Prevalencia de fluorosis dental en escolares de Santafé de Bogotá. Universidad el Bosque División de Postgrados. Santafé de Bogotá; 1999.

23. Kelsey JL, Douglas TH. Methods in observational epidemiology. N.Y. Oxford University Press, 1986 Cap. 10 (Cross-Sectional another types of studies) p.244-257.

24. Pagano M, Gauvreau K, Principles of Biostatistics. Duxbury Press. Belmont p. 472.

25. Jackson RD, Kelly SA, Katz B, Brizendine E, Stookey GK. Dental fluorosis in children residing in communities with different water fluoride levels: 33-month follow-up. Ped Dent 1999; 21: 248 - 254

26. Kramer HC, Bloch DA. Kappa coefficients in epidemiology: An appraisal of a reappraisal. J Clin. Epidemiol; 41: 959 - 968.

27. Akpata ES, Fakiha Z, Khan N. Dental fluorosis in 12-15-year-old rural children exposed to fluorides from web drinking water in the Hail region of Saudi Arabia. Community Dent Oral Epidemiol 1997; 25: 324 $-327$.

28. Microsoft Excell 5.0, Microsoft Corporation 1997.

29. Programa Epi-info 6.2. OMS.
30. Stata corp 1999. Stata statistical software. Release 6.0 College station, T.X: Stata Corporation.

31. Saunders BD, Trapp RG. Bioestadísitica Médica. Manual Moderno. México; 1997.

32. Hiller KA, Wilfart G, Scmalz G. Developmental enamel defects in children with different fluoride supplementation - Follow-up study. Caries Res 1998; 32:405 - 411.

33. Angmar-Mansson B. Whitford GM. Enviromental and physiologycal factors affecting dental fluorosis. J Dent Res 1990; 69: 706 - 713.

34. Kumar J, Swango P, Haley V, Green E. Intra-oral distribution of dental fluorosis in Newburg and Kingston. J Dent Res 2000; 79: 1508 1513.

35. Vallejo D, Ortega O. González F. Niveles de fluoruros en la sal de consumo humano de la ciudad de Bucaramanga. [Tesis de Grado]. Bucaramanga: Universidad Santo Tomás; 2002.

\section{2}

